\title{
Vivenciando o processo de municipalização do SUS no município de Juazeiro do Norte (CE)
}

\author{
Living the process of the municipalization of SUS \\ in Juazeiro do Norte, Ceará State
}

M ariaWanderleya de Lavor Coriolano ${ }^{1}$

GrayceAlencar Albuquerque ${ }^{2}$

Natércia de Sousa Araújo ${ }^{2}$

$M$ arcelo Alves de Oliveira ${ }^{2}$

$M$ arinus de $M$ oraes Lima ${ }^{3}$

${ }^{1}$ Universidade Federal de Pernambuco, Centro de Ciências da Saúde. Av. Prof M oraes Rego s/n, Prédio dasPós-Graduações do CCS/ 10 andar, Cidade Universitária. 50670-420 Recife PE.

wandenf@yahoo.com.br 2 Secretaria M unicipal de Saúde deJ uazeiro do N orte. ${ }^{3}$ Hospital Geral de Fortaleza.
Abstract The reorganization of the Brazilian health system, the principle of the decentralization and municipalization of the health services has demanded a deeper knowledge about its reality for people's quality of life. Theaim of thisstudy is to observe the organizational strategies of the health care in the city of Juazeiro do N orte, located in the state of Ceará, through a descriptive field research, with a quantitative-qualitative approach, extracting indicators of the life conditions of populations and semi-structured interviews held with main management demands on the field of SU S. The results proved a reality similar to most Brazilian towns, marked by the lack of recourses in the sector, health problems of different complexities to resolve, demonstrating a reality with certain details, such as: the existence of a bigmigration population assisted by the health system. Thus, it is necessary to analyze SU S with further objectivity, by understanding it as a process in permanent construction, taking into account the several difficulties to be faced.

Key words SU S, M unicipalization, M anagement
Resumo Com a reorganização do sistema desaúde brasileiro e o princípio da descentralização e municipalização dos serviços de saúde, torna-se de caráter relevanteum conhecimento maisaprofundado sobrea realidadeda munici palização para a qualidade de vida das pessoas. 0 objetivo do estudo é observar as estratégias organizacionais da atenção à saúde no município de Juazeiro do N orte (CE), através de uma pesquisa de campo descritiva, com abordagem quantiqualitativa, extraindo indicadores das condições de vida da população, e entrevistas semiestruturadas, realizadas com principais instâncias de gerência no âmbito do SU S. Os resultados evidenciaram uma realidade semelhante à maioria dos municípios brasileiros, marcada pela insuficiência de recursos no setor, problemas de saúde de diferentes complexidades resolutivas, demonstrando uma realidade com determinadas particularidades, tais como a existência de uma grande população flutuante, atendida pelo sistema de saúde. D esta forma, há de se analisar o SU S com maior objetividade, entendendo-o como processo em construção permanente, haja vista as inúmeras dificuldades a serem enfrentadas.

Palavras-chave SUS, M unicipalização, Gestão 
Introdução

A implantação formal do SistemaÚ nico de Saúde e sua estrutura nos dias atuais em distintos e particulares contextos territoriais ( país, estado, município, comunidades, entre outros) teve como marco fundamental a VIII Conferência N acional deSaúde, na qual se tornou evidente a necessidade de reorganização e reformulação de práticas e percepções envolvendo a saúde da população.

Sob o desenho do Sistema Único de Saúde, emergem vários princípios de organização e gestão da assistência, dentre os quais se insere a municipalização da saúde, entendida como uma estratégia a ser operacionalizada no sentido de que, para atingir o grau de mudanças necessárias em uma determinada real idade local, há a necessidadedeser desenvolvida a capacidade de coordenar os recursos disponíveis, tornando-se fundamental a participação de todos os atores sociais.

A orientação voltada para a descentralização na programação e planejamento em saúde exige informações sobre o perfil de morbimortalidade, os fatores de risco e seus determinantes, as características demográficas, informações sobre os serviços, bem como a disponibilidade de recursos humanos, de infraestrutura efinanceiros ${ }^{1}$.

Nesta lógica, conhecer einterpretar o Sistema Único de Saúde como estratégia operacional da atenção à saúde constitui uma tarefa complexa e um tanto desafiadora, devido à existência de inúmeras variáveis que precisam ser analisadas e interpretadas neste processo histórico multideterminado ${ }^{2,3}$. No caso, a construção do diagnóstico da situação de saúde do município de Juazeiro do N orte, o qual contempla indicadores intersetoriais, indicadores de saúde nos três níveis de atenção eentrevistas com instâncias de gerência econtrole no âmbito do setor de saúde, é apenas uma das variáveis que podem e devem ser estudadas, objetivando obter conhecimentos acerca da situação do SUS em um dado território.

Neste sentido, o trabalho epidemiológico é valioso para organizar egerir sistemas, sendo que a massa de todas as informações levantadas subsidiará o planejamento de ações a serem desenvolvidas com a população, levan do em conta suas individualidades e necessidades mais urgentes.

Diante desta perspectiva, foi consolidado um diagnóstico de saúde do município de Juazeiro do Norte (CE), levando em conta os principais indicadores de saúde da população, bem como percepções e conhecimentos das principais instâncias de gerência no âmbito do SUS, visando oferecer subsídios para a construção de políticas de saúde mais equânimes e eficazes frente às ne cessidades encontradas na população, além de mostrar como vem se dando o processo de municipalização do SUS, suas potencialidades, conquistas e desafios a serem perseguidos.

\section{Objetivos}

\section{Objetivo geral}

Observar as estratégias organizacionais da atenção à saúde no município de Juazeiro do Norte (CE).

\section{Objetivos específicos}

- Construir o diagnóstico da situação de saúde do município de Juazei ro do Norte (CE);

- Conhecer os principais indicadores de saúde do município;

- Entrevistar atores responsáveis por ações desaúde operacionalizadas no nível municipal e microrregional, analisando as suas percepções quanto ao Sistema Ú nico de Saúde;

- Conhecer os instrumentos de gestão utilizados no acompanhamento e avaliação das ações em saúde.

\section{M etodologia}

0 desenho do estudo é do tipo descritivo, ecológico, de abordagem quantiqualitativa ${ }^{4}$.

Foi desenvolvido no município de Juazeiro do Norte (CE), situado na região do Cariri, a 527 quilômetros de Fortaleza ${ }^{5}$, no período compreendido entre os dias 15 de março e 24 de abril de 2004, constando das seguintes etapas: levantamento de dados; revisão bibliográfica; realização de entrevistas; organização, sel eção e interpretação das informações na construção do diagnóstico de saúde e organização e interpretação das informações recolhidas a partir das entrevistas.

\section{Resultados}

Indicadores de saúde

Com os resultados da primeira etapa de investigação, foram extraídos alguns indicadores da atenção para desvelar informações prévias concernentes ao estado de saúde da população do município de Juazeiro do Norte (CE), com 
informações dispostas no plano municipal de saúde ${ }^{5,6}$, elaborado a partir do trabalho diário desenvolvido pelo Programa de Saúde da Família, dados de mortalidade dispostos no Sistema Nacional deM ortalidade (SIM ), dando conta das principais causas de morte das pessoas, para, assim, se dar o processo de planejamento e elaboração de estratégias voltadas às necessidades da população.

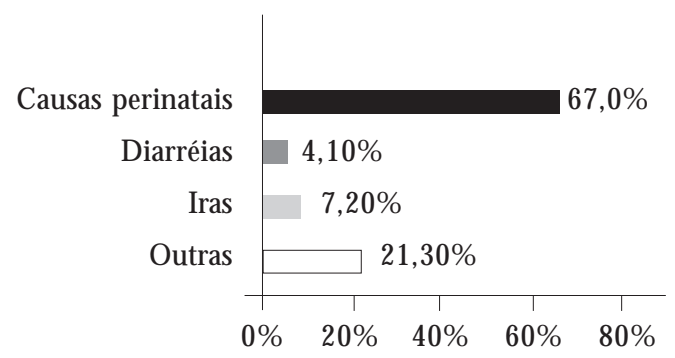

Gráfico 1. Causas de mortalidade infantil no ano de 2000. SINASC, 2000.

Fonte: Plano municipal de saúde, 2001.
O Gráfico 1 demonstra que as causas neonatais, por serem mais complexas edecontrolebem mais difícil, sendo suas principais causas problemas perinatais, anomalias congênitas ou de origem genética, estão em um patamar mais elevado $(67 \%)$ do que os óbitos pós-neonatais, cujas causas mais comuns são a diarréia, infecções respiratórias agudas (IRAS) e doenças infecciosas. Estas últimas, por sua vez, necessitam de medidas como saneamento básico, atendimentos de pré natal, com ênfase desde a primeira consulta no aleitamento materno exclusivo até os seis meses, consultas de puericultura, imunizações, utilização da terapia de reidratação oral e tratamento adequado das IRAS para obter impacto positivo sobre estes problemas.

Os dados obtidos através da interpretação do Gráfico 2 revelam que a principal causa de mortalidade geral deve-se às doenças cardiovasculares, correspondendo estas a $16,6 \%$ do total de óbitos, tendo as mesmas aumentado durante o período de 1997 a 2000. Em seguida, aparecem os achados anormais em exames clínicos e laboratoriais, com $16 \%$ do total de óbitos, apesar de sofrerem uma diminuição relativa durante 0 mesmo período. Em terceiro lugar, verifica-se a ocorrência de causas perinatais, perfazendo um

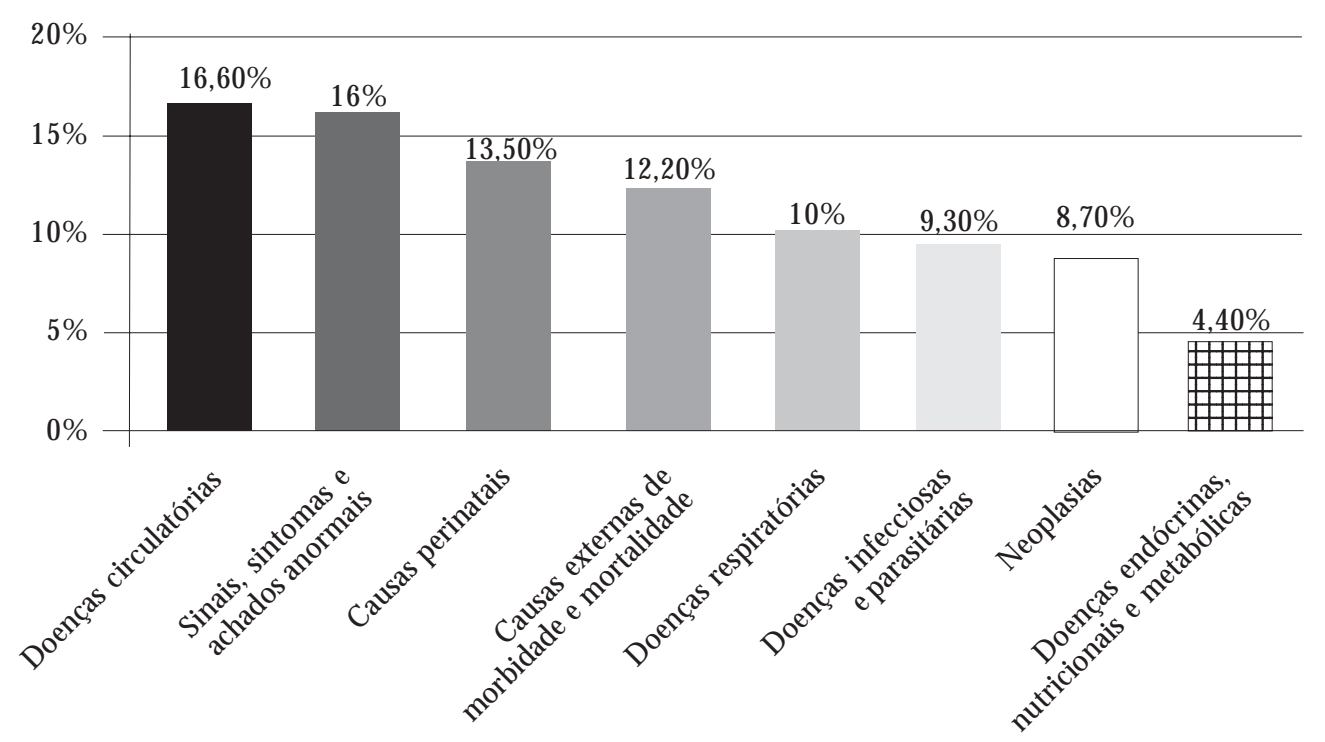

Gráfico 2. M ortalidade por grupo de causas de 1997 a 2000. DATASUS, 2000. 
total de $13,5 \%$, tendo oscilado durante o período estudado, com aumentos entre os anos de $1997 \mathrm{e}$ 1999 e diminuição entre 1999 e 2000. A parecem também como causas relevantes de mortalidade as causas externas, doenças respiratórias, doenças infecciosas e parasitárias, neoplasias e doenças endócrinas, nutricionais e metabólicas.

\section{Entrevistas realizadas com instâncias degestão}

\section{Programa de Saúde da Família (PSF)}

Atualmente, a atenção básica tem como estratégia operacional o Programa de Saúde da Família (PSF), cuja proposta concentra-se em integrar e organizar atividades de atenção à saúde em um dado território, numa visão hierárquica de comunicação com os níveis secundários e terciários de atenção à saúde, quando ocorre o defrontamento com problemas de resoluções mais complexas.

N esta dimensão, o PSF deve atuar na Unidade de Saúde da Família e nos domicílios da população, com uma visão integral da saúde dos indivíduos, estando vinculado à rede de serviços, de forma que se garanta atenção integral aos indivíduos e famílias e que sejam asseguradas a referência e a contrarreferência para clínicas e serviços de maior complexidade sempre que o estado de saúde da pessoa assim o exigi r $^{7,8}$.

A equipedo PSF é composta, no mínimo, por um médico, um enfermeiro, um auxiliar deenfermagem e de quatro a seis agentes comunitários de saúde (ACS), enquanto outros profissionais dentistas, assistentes sociais e psicólogos - poderão ser incorporados às equipes, visando aumentar a sua efetividade junto à clientela.

Assim, o cuidado da saúde da família objetiva a promoção da saúde através da mudança, propondo ajudar as famílias a criarem novas formas de interação para lidar com a saúde e contra agressores ${ }^{8}$.

De acordo com a Coordenadora do Programa de Saúde da Família do município de Juazeiro do Norte (CE), o município possui 42 Equipes de Saúde da Família (ESF), distribuídas na zona urbana e rural, cada uma atendendo em média 4.200 habitantes por área, sendo consideradas suficientes para atender os problemas de saúde enfrentados pelos indivíduos adstritos ao território coberto pela equipe responsável.

As principais atribuições e competências da coordenação do PSF consistem em acompanhar o desempenho das atividades executadas por cada equipe, por meio de avaliações mensais dos indicadores de saúde, através de relatórios produzidos por cada equipe, reuniões na Secretaria de Saúde e visitas às Unidades de Saúde. Além disso, planeja estratégias para ações de saúde junto às equipes, atualiza as informações do programa e promove a educação continuada para as ESF.

A entrevistada refere que, após a introdução dos trabalhos do PSF, foram observadas melhorias nos indicadores de saúde da atenção básica; porém, ressalta a existência de fal has, tais como a não cobertura de $100 \%$ da população, gerando uma grande demanda espontânea; o baixo nível sociocultural da população, dificultando o entendimento dos objetivos do programa, ea falta de profissionais com perfil de saúde da família.

Portanto, convém destacar que o programa, por si só, não garante uma efetiva resolução dos problemas de saúde, sendo observado que alguns pré requisitos, tais como a percepção e participação da população junto ao programa, o empenho equalificação dos profissionais de saúde e uma verdadeira articulação com o restante da rede, são indispensáveis para garantir o sucesso da atenção básica.

\section{Entrevista \\ com diretor do hospital de referência}

A criação econsolidação do Sistema Único de Saúde representa, em síntese, uma profunda modificação do arcabouço estrutural do setor desaúde no Brasil, o que évisualizado através do conteúdo demográfico de seus princípios, entre os quais se pode citar a regionalização dos serviços de saúde?.

A regionalização se expressa como uma nova forma de organização, caracterizada pela articulação entre os municípios de uma determinada região, constituindo uma redeúnica eintegrada, que tem como objetivos otimizar os recursos, melhorar a qualidade dos serviços ligados ao SUS nesses municípios e garantir 0 acesso aos mesmos. Desta forma, caso um município não apresente uma demanda maior para um certo serviço de média ou alta complexidade, outro município da rede que disponha de tal serviço pode oferecê-lo, garantindo 0 acesso.

A 21ạ Célula Regional de Saúde, localizada no Juazeiro do Norte, possui alguns hospitais de referência em serviços especializados dealta complexidade.

Segundo o diretor entrevistado, o hospital sob sua responsabilidade é um polo de referência 
macrorregional, que tem como especialidades a neurocirurgia, captação de órgãos para transplantes e a hemodiálise. Além disso, é hospitalescola da Faculdade de M edicina de Barbalha, entre outras atividades.

Os principais problemas vivenciados diretamente pelo hospital incluem baixa remuneração do SUS, aumento do preço dosinsumose limitações do número de atendimentos de alta complexidade.

A pactuação de compra de serviços entre os municípios e o hospital é feita através de PPI, pactuada na bipartite microrregional.

O setor de controle e avaliação é incipiente, sendo o controle da assistência prestadora feito pela qualificação e treinamento sistemático dos profissionais. $\mathrm{N}$ ão há sistema de ouvidoria.

Por fim, na concepção do diretor entrevistado, "Sem o SUS o povo morreria à míngua, é o único apoio de saúde para a população pobre, é a solução".

\section{Secretaria M unicipal de Saúde}

Em entrevista realizada com o Secretário de Saúde do município de Juazeiro do Norte, foi relatado que percebe o SUS como o maior plano de saúde do Brasil, não tendo atingido sua plenitude eacredita que nunca atingi rá devido ao número elevado deusuários, visto queele se destina a todos os brasileiros, tanto os que podem usufruir da assistência médico supletiva, como aqueles que não podem pagar. Contudo, a busca pelo ideal deve ser perseguida continuamente, sendo possível notar uma melhoria gradativa do sistema de saúde desde sua implantação até o momento atual.

Com relação ao fortalecimento da municipalização, reconhece muitas vantagens trazidas pelo SUS, no sentido de que os municípios ficaram mais preparados para assistir os seus munícipes. Outro fato importante diz respeito ao financiamento do setor, que se encontra "amarrado", ou seja, dividido em porcentagens para cada área de investimentos.

Referiu, ainda, queo município está habilitado na gestão plena ampliada, na qual a Secretaria de Saúde administra os valores destinados à saúde, ficando a sua aprovação sob a responsabilidade do Conselho Municipal de Saúde e do Executivo Municipal.

Quanto aos problemas de saúde enfrentados pelo município, os principais são doenças infectocontagiosas, incluindo a tuberculose e a hanseníase, além das doenças sazonais, tais como as diarréias e a dengue, sendo ambas de responsabilidade da população e do governo, e os recursos, que não são suficientes para o setor.

As ações contidas no plano municipal de saúde e a sua execução são prestadas junto ao Conselho Municipal de Saúde, sendo uma função obrigatória em prazo trimestral; entretanto, a prestação decontas érealizada mensal mente para não acumular trabalhos.

0 orçamento financeiro tem que ser aprovado pelo CM S para ter validade. As contas são previamente avaliadas por uma comissão especializada; depois da análise, as interpretações são repassadas ao conselho e, posteriormente, à plenária. Existe também uma central de contabilidade destinada apenas para o setor de saúde, localizada na prefeitura, sendo a prestação de contas realizada trimestralmente, indo em seguida para o CMS, câmara de vereadores, Ministério Público e Tribunal de Contas dos municípios.

Dentro da estrutura organizacional da secretaria, existe um setor de controle e avaliação, o qual é inerente às atividades e competências da secretaria, precisando-se desse serviço devido à complexidade das informações requeridas e prazos que precisam ser cumpridos. Existe, também, uma equipe responsável pela auditoria; no entanto, não se encontra totalmente estruturada para atender às várias funções. Assim, enquanto o setor de controle e avaliação informa e analisa, o de auditoria fiscaliza. Já o serviço de auditoria no município funciona através de um disque denúncia, não havendo um sistema estruturado.

A qualidade da assistência nos três níveis de aten ção (primária, secundária eterciária) baseiase nos indicadores de saúde: mortalidade infantil, cobertura vacinal, mortalidade materna, entre outros, os quais têm melhorado consideravelmente, em virtude do aumento no número de equipes de PSF, embora ainda tenha sua atuação prejudicada tanto pelas questões culturais da população (hospitalocêntrica, curativa), quanto pela falta de compromisso e qualificação profissional. Além disso, a qualidade da assistência também pode ser avaliada "pelo que não se faz", ou seja, observa-se uma menor demanda aos serviços de média e alta complexidade, demonstrando-se uma mai or eficácia da atenção básica.

\section{Conselhos Municipais de Saúde}

Os conselhos de saúde são os órgãos de controle do SUS pela sociedade nos níveis municipais, estadual e federal, tendo sido criados para permitir a interferência da população na gestão 
de saúde, defendendo os interesses das coletividades para que estes sejam atendidos pelas ações governamentais ${ }^{10}$.

Os consel hos funcionam, portanto, como órgão dirigente, cujos membros têm caráter permanente e deliberativo, devendo funcionar e tomar decisões regularmente, exercendo ações de acompanhamento, controle e fiscalização das políticas de saúde, além de propor correções e aperfeiçoamentos em seu rumo.

No município de Juazeiro do Norte (CE), o consel ho de saúde é composto por 24 membros, dispostos da seguinte forma: $25 \%$ são membros do governo, $25 \%$, prestadores de serviço e profissionais de saúde e $50 \%$, usuários, compreendendo associações e representantes de entidades de classe, os quais se reúnem mensalmente para tratar sobre assuntos relacionados ao funcionamento e gestão dos serviços de saúde, avaliar o relatório de gestão, pactuação de indicadores da atenção básica, PPI, plano municipal de saúde e setor de auditoria hospitalar e ambulatorial.

Em entrevista realizada com um membro do conselho, foi informado que o consel ho controla o cumprimento das ações planejadas pelo Executivo Municipal através de reuniões mensais de avaliação, nas quais são discutidas as principais decisões tomadas pelo conselho, as quais são defendidas em resoluções ou atos normativos.

Quanto à discussão e homologação do plano municipal de saúde (PMS) pelo Conselho Municipal de Saúde, foi relatado que o PMS é elaborado ediscutido por uma equipetécnica da Secretaria Municipal de Saúde, juntamente com o conselho, sendo votado e aprovado pelo Conselho Municipal de Saúde, o qual faz o acompanhamento quanto à execução do que foi estabe lecido no plano municipal de saúde.

A prestação de contas de atividades realizadas pelo CM S à população é realizada por meio de relatórios anuais, divulgações de resoluções e decisões na imprensa local, além da disseminação de informações pelos representantes da sociedade civil organizada àqueles que Ihes asseguraram o direito de representação junto ao CMS.

Ao se questionar a avaliação da assistência prestada ao usuário do município, o conselheiro entrevistado ressaltou que, apesar de existirem dificuldades, tais como a existência de uma população flutuante de um milhão de romeiros por ano; a falta de recursos para manutenção do sistema e a falta de compromisso dos profissionais de saúde, o conselho mantém presença nas suas atividades, como também em setores da Secretaria de Saúde, exercendo controle e avaliação através de visitas às Unidades de Saúde e discussões mensais com os diversos membros que 0 compõem, e as decisões tomadas pelo órgão são cumpridas pela Secretaria de Saúde para serem realizadas.

\section{Células regionais de saúde}

As atuais células regionais de saúde, anteriormente denominadas microrregiões de saúde, são definidas como espaços territoriais compostos por um conjunto de municípios, formadas num processo espontâneo e natural de integração e interdependência, constituindo espaços de negociação, articulação, coordenação e organização dos serviços entreos municípios, com vontade política unificada e capacidade de propor soluções para problemas enfrentados pela população dessa aérea geográfica, no sentido de garantir 0 atendimento das necessidades sentidas no âmbito da saúde?

Assim, no propósito de fortalecer a descentralização da saúde sem ocasionar custos onerosos ao sistema de saúde, a lógica das microrregiões visa regionalizar os serviços de maior complexidade, tornando-os mais acessíveis àqueles necessitados, de forma a facilitar o tratamento, cura e reabilitação, que não podem ser resolvidos na rede básica de atenção à saúde. Ao mesmo tempo, ocorrerá uma racionalização de recursos escassos, viabilizando a implantação de sistemas integrados de serviços de saúde, substituindo a fragmentação dos serviços, que é comprovadamenteineficaz.

Com os papéis inerentes à Célula Regional de Saúde, participando do processo de descentralização e cooperação entre gestores municipais e a esfera estadual, torna-se possível e pertinente a priorização de al guns tipos de especialidades mé dicas, conforme as condições de vida das populações adscritas a cada município, objetivando organizar efortalecer o desenvolvimento do SU Ssob uma lógica organizacional sustentada através da pactuação entreos atores envolvidos, estabelecendo-se o processo de distribuição dos serviços e recursos financeiros, sob o controle das Comissões Intergestoras Bipartites, que constituem fóruns de negociação entre municípios e Estado.

Segundo informação obtidas através de entrevista com a gerente da 21a Célula Regional deSaúde (composta pelosmunicípios de Barbal ha, Caririaçu, Granjeiro, Jardim, Juazeiro do N ortee M issão Velha), foi definido queo papel da célula regional de saúdeno contexto SU S consisteem fortalecer os sistemas municipais de saúde e apoiar e 
assessorar na lógica organizacional das microrregiões de saúde, de formaqueos municípiosagregados se unam e se articulem cooperativamente.

Quanto aos maiores problemas que a célula enfrenta, a mesma relata serem: (1) 0 atendimento hospitalar (devido à demanda elevada e o não cumprimento da Programação Pactuada I ntegrada-PPI); (2) a atenção básica (qualidadee quantidade), pois não se tem uma cobertura de PSF que atinja 100\% (por exemplo, Juazeiro do N ortepossui uma cobertura de $60 \%$ ); (3) carência de profissionais médicos para compor as equipes de PSF; (4) endemias, devido ao não envolvimento da população; (5) a escassez de recursos financeiros e (6) a qualificação dos profissionais de saúde.

Em relação ao financiamento pelo SUS, as três instâncias governamentais (União, Estado e Município) participam do financiamento da atenção primária; a NOAS prevêqueo município seja o responsável pelo mesmo, enquanto o Estado contribui com a assistência farmacêutica, vigilância sanitária e epidemiológica, além do nível federal contribuir com o Piso de Atenção Básica (PAB) e outros incentivos ao PSF.

O financiamento da atenção secundária, constituída por hospitais que tenham as especialidades prioritárias de pediatria, gineco-obstetrícia, cirúrgica geral, psiquiatria, traumato-ortopedia, urgência eemergência, rede de consultas especializadas, éfornecido o maior percentual pelo nível federal em municípios habilitados na gestão plena, enquanto aqueles habilitados na gestão básica recebem a maior parte dos incentivos provenientes do Estado. Os cálculos processados para o fornecimento deincentivos financeirosécalculado per capita, assim como os incentivos para a atenção básica.

No caso da aten ção terciária, o nível federal é responsável pela totalidade de recursos fornecidos, sendo que o financiamento nos três níveis de atenção não passa pela célula e os municípios recebem os recursos diretamente.

Ao se questionar sobre as instâncias democráticas de discussão e deliberação dos problemas de saúde, a gerente entrevistada reconheceu a fundamental importância dos conselhos municipais de saúde, bem como a participação das bipartites microrregionais quanto à apreciação de questões inerentes ao sistema de saúde, passando problemas que excedem sua capacidade resolutiva à comissão bipartite do Estado. M encionou ainda a tentativa de criar comissões que passam pelo nível da célula para investigar problemas (por exemplo, maus-tratos) junto às populações acometidas.
Ainda foi relatado que 0 acompanhamento da célula regional de saúde sobre as metas preestabelecidas nas estratégias de saúde baseia-se no plano estratégico, anualmente elaborado, além de ser feito o monitoramento por meio de outros instrumentos, tais como o Pacto da Atenção Básica, sendo cada município avaliado individualmente com base nos indicadores atingidos, comparando-os às metas fixadas nas agendas de saúde e planos municipais de saúde.

\section{Conclusão}

0 reconhecimento da necessidade de elaboração de planos locais para a saúde de suas populações constitui um requisito importante e crucial para o desenvolvimento dos diversos territórios.

Diante desse fato, o município de Juazeiro do Norte, lócus do presente estudo, exprime uma realidade semelhante à maioria dos municípios brasileiros, marcada pela insuficiência de recursos no setor, problemas de saúde de diferentes complexidades resolutivas, demonstrando uma realidadecom determinadas particularidades, tais como a existência de uma grande população flutuante, atendida pelo sistema de saúde, enquanto os recursos destinados à manutenção deste são calculados de acordo com a população fixa, gerando sobrecarga no atendimento aos problemas de saúde que necessitam ser enfrentados.

É interessante observar que as informações obtidas na realização de entrevistas com as várias instâncias gerenciais são coerentes entre si. Todos os entrevistados reconheceram a importância dos princípios do SUS na sua efetivação, apesar da existência de fal has ainda vivenciadas, a exemplo da não cobertura de $100 \%$ da população pelas equipes de PSF, limitações do número de atendimentos de média e alta complexidade, baixa remuneração do SUS, falta de profissionais qualificados em saúde da família, visão da população quanto à saúde centrada no modelo médico, curativo, inexistência da contrarreferência, que garantiria os princípios da universalidade, integralidadee intersetorialidade.

Por tudo isso, há de se analisar o SUS com maior objetividade, entendendo-o como processo em construção permanentequevisa, a médio e longo prazos, a uma mudança do paradigma de atenção à saúde e a uma busca de um sistema de saúde eficaz, eficiente, de qualidade equitativo. 


\section{Colaboradores}

MWL Coriolano, GA Albuquerque, NS Araújo, M A OliveiraeM M Lima participaram igualmente de todas as etapas da elaboração do artigo.

\section{Referências}

1. Bertolozzi MR, Fracolli LA. O perfil epidemiológico na prática do enfermeiro no PSF. In: M inistério da Saúde. M anual de Enfermagem. Brasília, M inistério da Saúde; 2001.

2. Ciampone MHT, Peduzzi M. Planejamento Estratégico como Instrumento de Gestão e Assistência. In: Ministério da Saúde. M anual de Enfermagem. Braślia: M inistério da Saúde; 2001. p. 23-28.

3. Rouquayrol MZ, Almeida Filho N. Epidemiologia e saúde. 5a ed. São Paulo: M edsi; 1999.

4. Oliveira SL. Tratado de metodologia científica: projetos de pesquisa, TGI, TCC, monografias, dissertações e teses. 2a ed. São Paulo: Pioneira; 1997.

5. Ceará. Perfil Básico M unicipal do M unicípio Juazeiro do N orte. [site da Internet] Disponível em: http:// www.iplance.ce.gov.br

6. Brasil. Informações sobre Saúde. [site da Internet] Disponível em: http://www.datasus.gov.br

7. Zoboli ELCP, Martins CL, Fortes PAC. O Programa de Saúde da Família na busca da humanização e da ética na atenção à saúde. In: Ministério da Saúde. M anual de Enfermagem. Programa de Saúde da Família. Brasília: Ministério da Saúde; 2001.

8. Bousso RS, Angelo MA. Enfermagem e o cuidado na Saúde da Família. In: M inistério da Saúde. Manual de Enfermagem. Brasília: M inistério da Saúde; 2001. p. 18-22.

9. Ceará. M icrorregiões de Saúde: uma opção do Ceará. Fortaleza: SESA; 2000.

10. Brasil. Lei no 8.142, de 28 de dezembro de 1990. Dispõe sobre a participação da comunidade na gestão do SUS e sobre as transferências intergovernamentais de recursos financeiros na área da saúde e dá outras providências. Diário O ficial da União 1990; $31 \mathrm{dez}$.

Artigo apresentado em 27/07/2007

Aprovado em 14/12/2007

Versão final apresentada em 07/04/2008 